\title{
Acoustic Inversions from an Explosive Source of Opportunity in the ASIAEX2001-SCS Experiment
}

\author{
Ying-Tsong Lin ${ }^{1}$, Chi-Fang Chen ${ }^{1}$, James F. Lynch ${ }^{2}$, Nicholas Chotiros ${ }^{3}$, Arthur Newhall ${ }^{2}$, \\ Altan Turgut ${ }^{4}$, Steve Schock ${ }^{5}$, Ching-Sang Chiu ${ }^{6}$
}

\begin{abstract}
Acoustic inversions including source localization and geoacoustic inversion from an unexpected explosion in the ASIAEX2001-SCS experiment is presented. Horizontal beamforming is performed to find the source azimuthal direction and the water wave part of the modal dispersion curve is used to find the source range. The relation of the source depth and the charge weight has been obtained from the bubble pulse period. The localization result shows that the detonation occurred in the direction of east-north east and about $30 \mathrm{~km}$ distant from the receiver. To perform the bottom inverse, we use a broadband linear inverse technique employing the modal group velocity, which is inferred from the modal arrival time and source range. In the end, a four-layered bottom model is created, which is consistent with both our explosion data and independent source tow data. The resolution and variance of the estimate are also presented. In conclusion, this bottom model should be widely applicable for modeling broadband sound propagation in the ASIAEX2001-SCS experiment site.
\end{abstract}

Index Terms-source localization, geoacoustic inversion, ASIAEX.

\section{INTRODUCTION}

On of the South China Sen (SCS) component of the Asian Seas International Acoustics Experiment (ASIAEX) was to obtain a good geoacoustic model of the bottom in the experimental area. Having such a model is essential to understanding the propagation and scattering of acoustic signals, which was the main focus of the experiment. In order to obtain a bottom model, two experimental acoustic activities were carried out in ASIAEX: 1 ) a series of source tows, using a J-15-3 source to transmit signals in the $50-260 \mathrm{~Hz}$ range to a moored vertical and horizontal array receiver [1] and 2) chirp sonar surveys along several tracks in the ASIAEX SCS experimental region [2,3]. However, none of these activities was able to provide a low-frequency, horizontally propagating signal, such as one obtains from standard "SUS" (Source of Underwater Sound) charge surveys. Thus the bottom surveys only provided a high-resolution view down to the penetration depth of the

'National Taiwan University, Taipei, Taiwan

${ }^{2}$ Woods Hole Oceanographic Institution, MA, USA

${ }^{3}$ Office of Naval Research, Arlington, VA, USA

${ }^{4}$ Naval Research Laboratory, Washington, DC, USA

sUniversity of North Carolina, Chapel Hill, NC, USA

'Naval Postgraduate School, Monterey, CA, USA
$50 \mathrm{~Hz}$ sound produced by the J-15-3 source. However, it appears that local "dynamite fishing" activity has provided us with some of the low frequency explosive signals that we were not able to get permission for in the experiment. During the course of the ASIAEX SCS experiment in May 2001, we detected at least four such explosions. Two of the records of these explosions were clipped, as the explosions were obviously nearby and our receiver's dynamic range was set for much lower level signals. Of the remaining two signals, only one had a significant "ground wave" (sometimes called "precursor") component, and we will concentrate on this explosion for this paper. There is probably some further information to be gained from the other signals as well, but we will just choose the most straightforward one for analysis here.

\section{THE EXPERIMENT GEOMETRY}

In Figure 1, we show the acoustic components of the experiment which are of most interest for the present analyses. To the west is the location of the main receiver array for the experiment, the so-called "Shark" mooring (named for the "Shark of Science" insignia painted on the receiver's experimental tow-sled), deployed in $124 \mathrm{~m}$ water depth. This moored receiver array consisted of a 32 element bottom-lying horizontal line array (HLA) of $-480 \mathrm{~m}$ extent $(15 \mathrm{~m}$ element spacing) and a 16 element vertical line array (VLA) of $82 \mathrm{~m}$ extent, with mixed 3.75 and $7.5 \mathrm{~m}$ inter-element hydrophone spacing [4]. The extreme northernmost piece of equipment displayed in Figure 1 is the PANDA receiver. Unfortunately, the explosion of most inerest in this paper occurred at UTC 1838 on May 16th, when the PANDA

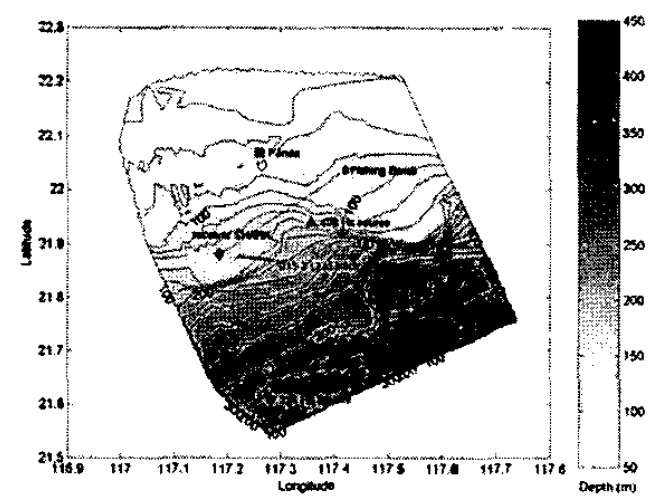

Figure 1: Locations of the various acoustics sources 
and receivers of most interest to the 2001 ASIAEX SCS bottom inversion efforts.

receiver was already retrieved, and so we lost the opportunity for the cross correlation distance estimate. Tuming to the sources, the one of most interest to us is the one farthest to the east in Figure 1, labeled "Fishing Bomb." The other source of interest is the red line labeled "J-15-3 LFM track," which shows the tow path which Turgut [1] considered in obtaining his bottom inverse results. There are two other source sites noted in Figure 1, marked " $224 \mathrm{~Hz}$ source" and " $400 \mathrm{~Hz}$ source." These are the two moored source sites used in the experiment. In doing the bottom inverses from the fishing bomb data, we should note that the source is at a distance from the receiver, and that the inverse that we derive will thus represent a range average of the bottom properties between source and receiver.

\section{ACOUSTIC INVERSION (I): SOURCE LOCALIZATION}

In Figure 2, we show the time series of the explosive source, so-called "source of opportunity", which was received at the VLA/HLA at UTC 1838, May 16, 2001. This signal displays an almost classic form, with a rumbling $\sim 8 \mathrm{~Hz}$ "ground wave" early arrival, a sharp explosion "water wave" component following it, and a fairly monochromatic "Airy phase" arrival of frequency $17.5 \mathrm{~Hz}$. This explosion has a peak SNR of $\sim 40 \mathrm{~dB}$, which gives us more than adequate power for our spectral analysis and other calculations. However, as we do not know the exact size of the explosive charge (as will be further discussed), we cannot perform transmission loss calculations and thus cannot estimate medium attenuation.

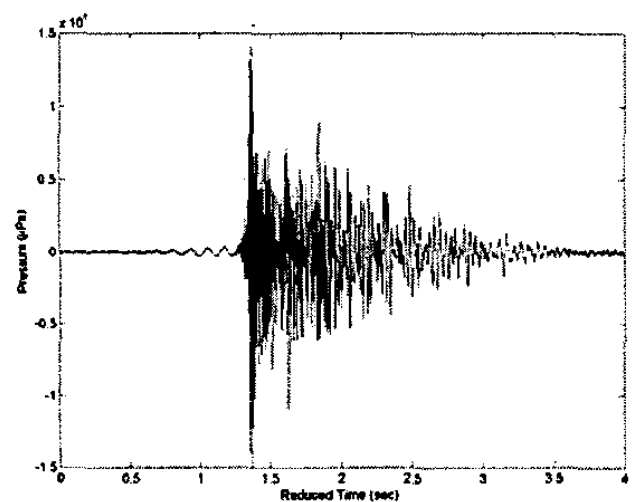

Figure 2: The explosive "source of opportunity" signal registered at the HLA/VLA receiver on May 16, 2001.

Though the time series representation of the explosion is interesting, the frequency versus time spectral view of the signal is more immediately useful in terms of the broadband inversion analysis we will be performing. A spectrogram of the detonation, obtained using the time-dependent Fourier analysis, is shown in Figure 3. One of the salient features of the spectrogram in Figure 3 is the bubble pulses, which are created by alternating cycles of expansion and compression of the hot explosive gases. From the time-frequency analysis, we can see that the time between the bubble pulses is $\sim 0.25$ seconds. This bubble pulse period may be used to estimate the depth/charge-weight ambiguity of the explosion. From the result, we see that for any reasonable sized "fishing explosion", which would probably be comprised of a few sticks of dynamite weighing a few kilograms at most, the source depth was probably rather shallow, i.e. less than 20 meters below the surface. This places it in the higher soundspeed region near the sea surface (see Figure 4) for the ASIAEX SCS experimental site. This is important acoustically, in that having a source in a higher speed region favors the excitation of high normal modes versus low normal modes. Indeed, we only see mode one faintly in our data, as shown in Figure 3.

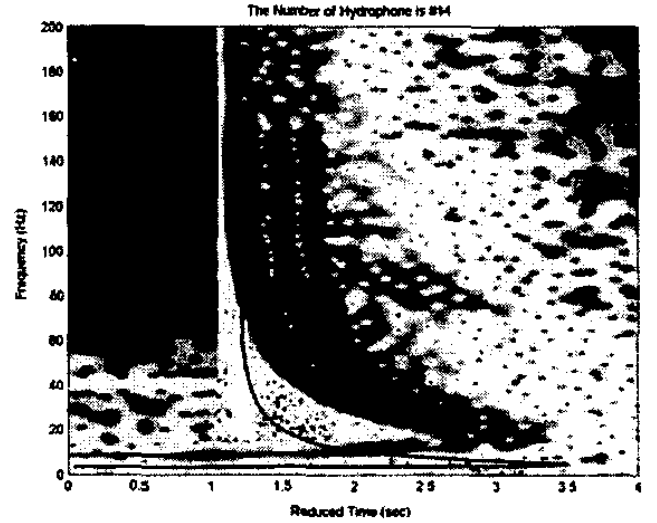

Figure 3: Spectrogram of the source of opportunity explosion shown in Figure 2, for a near bottom hydrophone. The second mode ground wave precursor at $\sim 8 \mathrm{~Hz}$ is clearly seen. One also notes the strong water wave arrival and two subsequent bubble pulses, particularly in the $100-200 \mathrm{~Hz}$ range. Mode one is seen faintly, with a weak $4 \mathrm{~Hz}$ precursor.

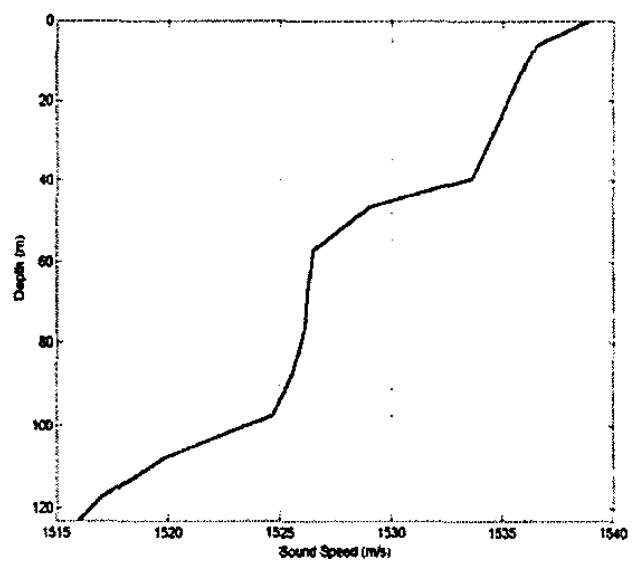

Figure 4. Soundspeed profile obtained from the temperature sensors on the VLA at the time the explosion was recorded. The explosive source was 
likely set off at a depth of $\sim 20 \mathrm{~m}$, i.e. in the higher soundspeed region of the profile.

Before we can extract a group velocity dispersion curve from the spectrogram, which is the basic input to our bottom inverse procedure, we need to obtain what the range is to the source. We also desire the bearing to the source, as this will tell us the bathymetry along the track to the source, which is especially important to know in a strongly range dependent environment like the ASIAEX SCS site. We now first look at the bearing to the explosion, estimated by steering the horizontal line array (HLA) to produce a maximum output at the source bearing. By doing simple time delay beamforming on the arrivals, and using the element positions shown below, it was found that the explosion came from the direction 35 degrees relative to the east. The error in this estimate, due to beam width, is on the order of a degree. In doing the ranging, we employed the well-known KRAKEN code [5] to compute theoretical "adiabatic" normal mode group velocity dispersion curves for candidate source ranges. This was done using Turgut's bottom model, water column soundspeed profile from the experimental site, and the bathymetry along the track to the explosion, shown in Figure 5. These theoretical curves were generated for a large variety of range possibilities, and then a best fit to the data shown in Figure 3 was obtained. The ranging results in a range of $29.3 \mathrm{~km}$ for the explosion. This estimate has error inherent in it due to errors in the bottom model, signal to noise ratio, etc. We will quantify that error in a later portion of this paper, where we discuss the error in the inversion for the bottom.

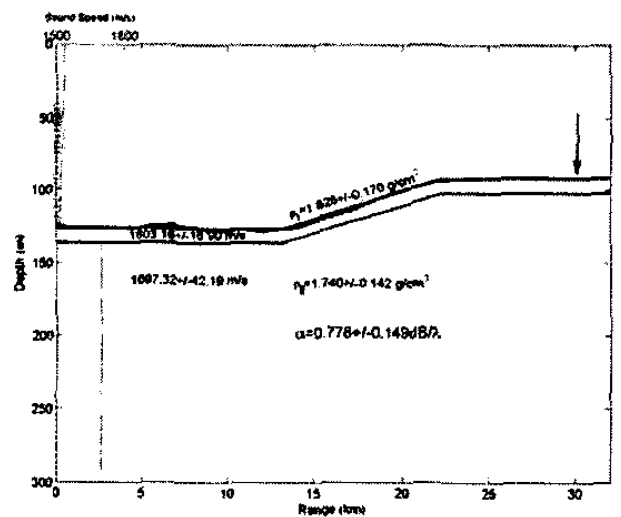

Figure 5: The bathymetry along the sourceto-receiver propagation path, along with the soundspeed profile at the receiver site. The vertical array hydrophone locations are shown on the $y$-axis. The estimated fishing explosion range is indicated by an arrow at $29.3 \mathrm{~km}$ distance from the receiver.

We should also discuss our claim that the strongest mode signal, which we are using as our prime bottom inverse data, is mode two rather than mode one, which is what one usually expects to see. This claim is supported by the facts that: 1) vertical array mode filtering (using the cleaner frequencies in the dispersion curve) clearly shows the mode two shape, 2) the strongest mode's cutoff is at $8 \mathrm{~Hz}$, indicative of mode two, whereas only a very weak signal at the lowest hydrophones is seen at $4 \mathrm{~Hz}$, indicative of mode one, and 3) using mode one in the source ranging estimate gives an unreasonable result of hundreds of kilometers.

\section{ACOUSTIC INVERSION (II): INVERSION FOR BOTTOM MODEL FROM THE EXPLOSIVE SIGNAL.}

Since we now have a distance estimate to the explosion, this is straightforward. We first define a new relative arrival-time from the arrival-time measurement by subtracting its first value, which is measured at the frequency $150 \mathrm{~Hz}$. Therefore, the range-averaged group velocity of the second mode is obtained by the following:

$$
\bar{v}_{g}(\omega)=\frac{R}{T}=\frac{R}{T_{\text {relative }}+R / \bar{v}_{g}\left(\omega_{0}\right)},
$$

where $R$ is the range estimate, $T$ is the travel time, $T_{\text {relative }}$ is the relative arrival time, and $\omega_{0}$ is the first frequency of the measurement, i.e. $150 \mathrm{~Hz}$ in our analysis. In the above formulation, the range-averaged modal group velocity at $\omega_{0}$ is estimated using the in situ sound speed in water and Turgut's bottom model. Performing this simple operation, we obtain the group velocity curves shown in Figure 6.

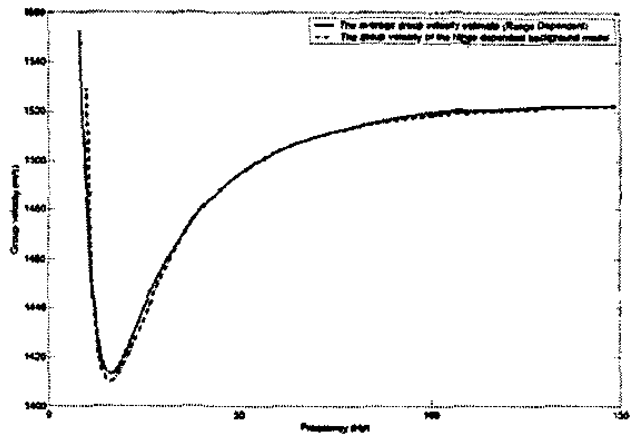

Figure 6: Group velocity of measured data versus that generated by the resolved range-dependent bottom model.

We now explain how to invert the group velocity for the bottom model. We notice that, for Turgut's bottom model[1], 50 $\mathrm{Hz}$ energy penetrates to about $50 \mathrm{~m}$ depth, so that using lower frequency data, we are justified in adjusting the sound speed below that depth to values which differ from Turgut's "half space". To extend Turgut's result, we find that we need to add two more layers below $50 \mathrm{~m}$ depth to the bottom model. We first consider the "basement" layer, the soundspeed of which is determined by the observed cutoff frequency of mode two. Since we know the horizontal wavenumber at $150 \mathrm{~Hz}$ from Turgut's bottom model, we can integrate the inverse of the group velocity curve from $150 \mathrm{~Hz}$ to $8 \mathrm{~Hz}$ (the cutoff frequency) to get the horizontal wavenumber at $8 \mathrm{~Hz}$. Let us just consider the range independent case, as the range dependent case is 
calculated same way. The value of the integration obtained is $k_{r}(8 \mathrm{~Hz}) \sim 2.039 \times 10^{-2} \mathrm{~m}^{-1}$. The soundspeed in the bottom halfspace thus should be $c=\omega / k_{r}$, which is $2340 \mathrm{~m} / \mathrm{s}$. However, just putting this halfspace directly under Turgut's model does not give a reasonable model to fit our (range independent) group velocity data. Thus, we add one more layer. The thickness of this new layer is found by noting that the Airy phase position in frequency is rather sensitive to its thickness we adjust this to give the correct position of the Airy phase, obtaining a $45 \mathrm{~m}$ thick layer. The soundspeed in this layer is found, by simple iteration of forward models, to be of the order of $1800-1900 \mathrm{~m} / \mathrm{s}$. Finally, we add a slight soundspeed gradient to all the layers in accord with Hamilton's regressions, to compensate for the overburden pressure (weight of the sediments above). The models thus generated are shown in Figure 7.

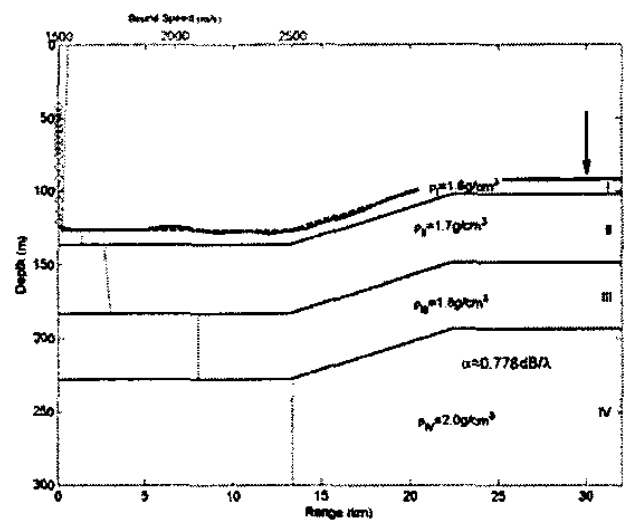

Figure 7: The bottom background models at the receiver position, in which the solid lines represent the range-dependent model.

Using the frequencies below $50 \mathrm{~Hz}$ as data and performing the perturbative inverse $[6,7]$, we find, rather interestingly, that our resolved model is not significantly improved by the procedure. Indeed, the $\Delta c$ produced just seems to add noise to the background model, and do not improve the mean square fit of the group velocity model to the group velocity measurement, shown in Figure 6. Thus is seems that we started with a good model to begin with. In a sense, this is not a surprise, because we initially adjusted two of the most important pieces of low frequency data, the cutoff frequency and the Airy phase, to get the bottom model. In addition, we also adjusted the soundspeed of layer number three iteratively beforehand, which is basically a nonlinear inverse procedure. We can thus say that, to the accuracy of the linear method, we have obtained a reasonable range-dependent bottom model.

Though our model was sufficiently good, the work done in creating the linear inverse estimates is far from wasted, in that one still needs the kernel of that inverse to determine the resolution and variance of the bottom models generated. Looking first at the vertical resolution of the estimate, we have Rajan and Lynch's formulation $[6,7]$ for the resolution kernel
$R\left(z, z_{0}\right)$, and we can reduce this depth dependent resolution kernel into a single number, the "resolution length," at any given $\mathrm{z}$ simply by reporting the width of $R\left(z, z_{0}\right)$. We do that in these analyses, obtaining the results shown in Figure 8. This resolution length physically represents how much of a "ve,tical average" of the medium properties the inverse produces at any given depth. We should note that this kernel just represents the resolution obtained from our broadband modal inverse; the improved resolution one gains from combining the chirp sonar, the J-15-3 tow source inverse, and the broadband source of opportunity inverse is not reported here, as the kernel for that combined inverse is much more difficult to construct.

We finally come to the variance in the estimate. We again adopt the methods used by Rajan, Lynch et al $[6,7]$. Assuming that the errors in each data point are uncorrelated and that the error variance is the same for all data (useful, though not always correct, assumptions) we obtain the variance shown in Figure 9.

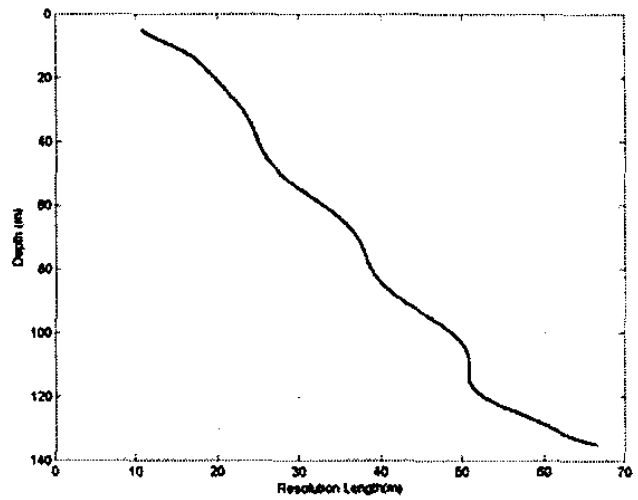

Figure 8: Resolution length versus depth for the range- dependent bottom model result.

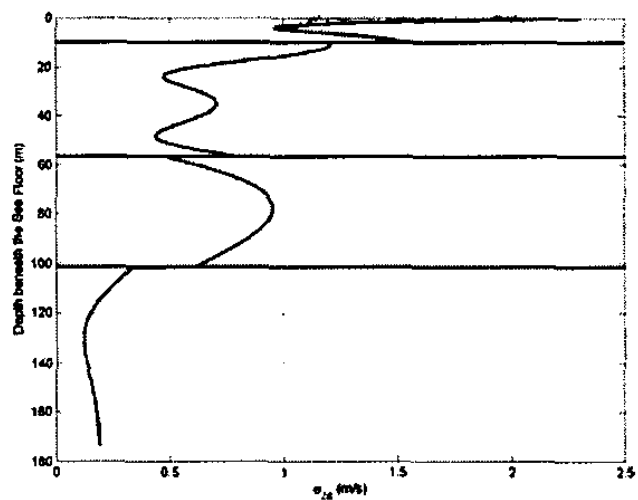

Figure 9: The variance of bottom soundspeed solution due to measurement inaccuracy. 


\section{Conclusions}

The results of this paper combine a priori data from chirp sonar and towed sources, which give better resolution than could have been obtained from the broadband inverse alone. The availability of a broadband source with good low frequency content was a major advantage, as it allowed us to probe deeper into the bottom than our other sources permitted. The lack of ranging information, while annoying, was not fatal, as we were able to estimate the range to the source using the higher frequency part of the dispersion curve. The lack of a source signature precluded our obtaining an attenuation estimate, and the lack of control of the source depth led to our not having as many modes available to invert as we would have had were we able to set off the charges at a desired depth.

The possibilities for improving our results are being investigated The use of the faint, incomplete, yet partially available mode one data seen in the lower hydrophones may give better resolution, and we should examine how much improvement this small amount of extra data gives. We should examine the resolution and variance of the combined inverse, versus the broadband data alone. The contribution of each type of data to the overall answer is an interesting study, and one which we would like to pursue. Finally, the fully range dependent, broadband adiabatic mode inverse needs to be further developed.

\section{ACKNOWLEDGMENT}

The authors must thank all of our sponsors, colleagues, and students for their support, collaboration, hard work, without which ASIAEX would not have succeeded. In particular, we will single out the ASIAEX coordinators, whose "behind the scenes" efforts cleared the path for the experiment, the captain and crew of the Fisheries Researcher I, which deployed and recovered our acoustic array, our super engineering and buoy deployment teams, which got us the data, G. Potty and J. Miller for their detailed comments on this manuscript and their wavelet calculations, and S. Wolf of NRL for his HLA localizations. This work was supported under ONR Grant numbers N0001498-1-0413, N00014-00-0931, and N00014-01-0772 and NSC (National Science Council, Taiwan, R.O.C.) Grant number NSC92-2611-E-002-005-CCS. This is WHOI contribution number 11009.

\section{REFERENCES}

[1] A.Turgut, et al., "Inversion of range-dependent geoacoustic properties in South China Sea ASIAEx01 experimental site," $J$. Acoust. Soc. Am., Vol 113, p. 2218, 2003.

[2] S. Schock, "A method for estimating the physical and acoustical properties of the seabed using chirp sonar data," Submitted to IEEE J. Oceanic Eng. Special Issue on the Asian Marginal Seas, 2003.

[3] S. Schock, "Remote prediction of physical and acoustical sediment properties in the South China Sea using chirp sonar data and the Biot model," Submitted to IEEE J. Oceanic Eng. Special Issue on the Asian Marginal Seas, 2003.

[4] A. Newhall, et al., Preliminary Acoustic and Oceanographic Observations $s$ form the ASIAEX 2001 South China Sea Experiment, Woods Hole Oceanographic Institution Technical Report, WHOI-2001-12, 2001.

[5] M.B. Porter, The KRAKEN normal mode program, Rep. SM-245, SACLANT Undersea Research Centre, La Spezia, Italy, 1991.
[6] S.D. Rajan, et al., "Perturbative inversion methods for obtaining bottom geoacoustic parameters in shallow water," J. Acoust. Soc. Am.., Vol 82, pp. 998-1017, 1988.

[7] J.F. Lynch, et al., "A comparison of broadband and narrow-band modal inversions for bottom geoacoustic properties at a site near Corpus Christi, Texas," J. Acoust. Soc. Am., Vol 89, pp. 648-665, 1988. 